\title{
EIGENVALUES FOR THE SEMI-CIRCULANT PRECONDITIONING OF ELLIPTIC OPERATORS WITH THE VARIABLE COEFFICIENTS
}

\author{
Hoi Sub Kim, Sang Dong Kim, And Yong Hun Lee
}

\begin{abstract}
We investigate the eigenvalues of the semi-circulant preconditioned matrix for the finite difference scheme corresponding to the secondorder elliptic operator with the variable coefficients given by $L_{v} u:=$ $-\Delta u+a(x, y) u_{x}+b(x, y) u_{y}+d(x, y) u$, where $a$ and $b$ are continuously differentiable functions and $d$ is a positive bounded function. The semicirculant preconditioning operator $L_{c} u$ is constructed by using the leading term of $L_{v} u$ plus the constant reaction term such that $L_{c} u:=-\Delta u+d_{c} u$. Using the field of values arguments, we show that the eigenvalues of the preconditioned matrix are clustered at some number. Some numerical evidences are also provided.
\end{abstract}

\section{Introduction}

Consider the second-order elliptic boundary value problem given by

$$
\begin{gathered}
L_{v} u:=-\Delta u+a(x, y) u_{x}+b(x, y) u_{y}+d(x, y) u=f \\
\text { in } \Omega:=(0,1) \times(0,1)
\end{gathered}
$$

with the homogeneous Dirichlet boundary condition

$$
u=0 \quad \text { on } \partial \Omega \text {. }
$$

The usual central finite-difference scheme [16] to find an approximated solution for $(1.1)$ is given by

$$
\begin{aligned}
& -\frac{1}{h_{2}^{2}}\left\{C_{k-1, j} u_{k-1, j}+A u_{k, j}+B_{k+1, j} u_{k+1, j}+\gamma_{k, j-1} u_{k, j-1}\right. \\
& \left.+\alpha_{k, j} u_{k, j}+\beta_{k, j+1} u_{k, j+1}\right\} \\
= & f_{k, j}, \quad 1 \leq k \leq m_{1}, 1 \leq j \leq m_{2},
\end{aligned}
$$

Received October 24, 2005.

2000 Mathematics Subject Classification. 15A12, 15A60, 65F15, 65 F35.

Key words and phrases. semi-circulant preconditioning, central finite difference, field of values. $0)$.

This work was supported by KOSEF C00006 which is same as (KRF R02-2004-000-10109- 
where

$$
\begin{gathered}
A=-2 \varphi^{2} \\
B_{k+1, j}=\varphi^{2}\left(1-\frac{a_{k, j} h_{1}}{2}\right), \quad C_{k-1, j}=\varphi^{2}\left(1+\frac{a_{k, j} h_{1}}{2}\right), \\
\alpha_{k, j}=-2-d_{k, j} h_{2}^{2}, \quad \beta_{k, j+1}=1-\frac{1}{2} b_{k, j} h_{2}, \quad \gamma_{k, j-1}=1+\frac{1}{2} b_{k, j} h_{2}
\end{gathered}
$$

and, $m_{1}$ and $m_{2}$ are positive integers such that

$$
h_{1}=\frac{1}{m_{1}+1}, h_{2}=\frac{1}{m_{2}+1}, \varphi=\frac{h_{2}}{h_{1}} .
$$

Here $u_{k, j}$ is the approximated value of $u(x, y)$ at the point $\left(x_{k}, y_{j}\right)$ and $g_{k, j}$ is the value of a $g(x, y)$ at the point $\left(x_{k}, y_{j}\right)$, where $g=f, a, b$ or $d$, and

$$
\left(x_{k}, y_{j}\right):=\left(k h_{1}, j h_{2}\right), \quad 1 \leq k \leq m_{1}, 1 \leq j \leq m_{2},
$$

are interior nodal points in the domain $\Omega$.

It is known that the semi-circulant preconditioner can be constructed by adding circulant elements to the matrix induced by finite difference discretization. In many literature, constructing semi-circulant and circulant preconditioner has been studied widely (see [1], [6], [7], [8], [9], [10], [11], [12] and [19]). These preconditioning strategy has been extended to HSS and PSS preconditioners (see [2] and [3]). Because the finite difference matrix $\hat{L}_{v}$ which represents the finite difference discretization corresponding to the operator $L_{v}$ on the left-hand side of (1.3) can not be represented as tensor product form, it may be required to set up a semi-circulant preconditioner matrix corresponding to $L_{c}$ given by

$$
L_{c} u:=-\Delta u+a_{c} u_{x}+b_{c} u_{y}+d_{c} u=f \quad \text { in } \Omega
$$

with zero boundary condition, where $a_{c}, b_{c}$ and $d_{c}$ are constants. We also note that the idea of optimal preconditioning in [17] is to consider a preconditioner by the leading term $-\Delta u+d_{c} u$, where $d_{c}$ is chosen as $d_{c}=\sqrt{a_{c}^{2}+b_{c}^{2}}$ so that $L_{c}$ remains an optimal preconditioning operator. Adopting this optimal choice, one may construct an optimal semi-circulant preconditioner for the case of variable coefficients. But in this paper, we choose

$$
d_{c}:=\min _{(x, y) \in \bar{\Omega}} d(x, y) \geq 0 \text { and } a_{c}=b_{c}=0 .
$$

The aim of this paper is to provide the distribution of eigenvalues and behavior of the extreme eigenvalues of the preconditioned matrix for the case that the coefficients $a(x, y)$ and $b(x, y)$ are continuously differentiable functions on $\Omega$, and $d(x, y)$ is a positive bounded function. These will be assumed throughout this paper. This analysis is based on the way setting up a semi-circulant preconditioner and analyzing the finite difference preconditioner in terms of coefficient functions even if the finite difference preconditioning is well known using the leading terms of the given operator (1.1) (for example, see [17], [18]). 
The usual central finite-difference scheme which approximates (1.8) with (1.9) is given by

$$
\begin{aligned}
& -\frac{1}{h_{2}^{2}}\left\{\hat{C} u_{k-1, j}+\hat{A} u_{k, j}+\hat{B} u_{k+1, j}+u_{k, j-1}+\hat{\alpha} u_{k, j}+u_{k, j+1}\right\} \\
= & f_{k, j}, \quad 1 \leq k \leq m_{1}, 1 \leq j \leq m_{2},
\end{aligned}
$$

where

$$
\hat{A}=-2 \varphi^{2}, \quad \hat{B}=\hat{C}=\varphi^{2},
$$

and

$$
\hat{\alpha}=-2-d_{c} h_{2}^{2}
$$

We assume that

$$
0 \leq \frac{\|a\|_{\infty} h_{1}}{2}<1, \quad 0 \leq \frac{\|b\|_{\infty} h_{2}}{2}<1
$$

Note that the coefficients in (1.9) trivially satisfy the above assumption (1.13). While the matrix $\hat{L}_{v}$ can not be expressed as a tensor product form, the matrix $\hat{L}_{c}$ which represents the finite difference discretization corresponding to the operator $L_{c}$ on the left-hand side of $(1.10)$ is easily described as a tensor product form:

$$
\hat{L}_{c}:=-\frac{1}{h_{2}^{2}}\left\{T_{2} \otimes I_{m_{1}}+I_{m_{2}} \otimes T_{1}\right\},
$$

where $I_{m_{k}}$ are the identity matrices with the size $m_{k}$, and $T_{1}$ and $T_{2}$ are the tridiagonal matrices of order $m_{1}$ and $m_{2}$ respectively. In particular,

$$
T_{1}=\operatorname{tridiag}[\hat{C}, \hat{A}, \hat{B}] \quad \text { and } \quad T_{2}=\operatorname{tridiag}[1, \hat{\alpha}, 1] .
$$

The semi-circulant preconditioner for (1.14) is given by

$$
\hat{S}_{c}:=-\frac{1}{h_{2}^{2}}\left\{\bar{C} \otimes I_{m_{1}}+I_{m_{2}} \otimes T_{1}\right\},
$$

where $\bar{C}$ is the circulant matrix corresponding to $T_{2}$ given by

$$
\bar{C}=\left(\begin{array}{cccccc}
\hat{\alpha} & 1 & 0 & \cdots & 0 & 1 \\
1 & \hat{\alpha} & 1 & 0 & \cdots & 0 \\
0 & 1 & \hat{\alpha} & 1 & 0 & \vdots \\
\vdots & \ddots & \ddots & \ddots & \ddots & 0 \\
0 & \cdots & 0 & 1 & \hat{\alpha} & 1 \\
1 & 0 & \cdots & 0 & 1 & \hat{\alpha}
\end{array}\right) .
$$

In this paper we will propose $\hat{S}_{c}$ in (1.16) as a semi-circulant preconditioner for $\hat{L}_{v}$ which is the matrix representation of $(1.3)$. The semi-circulant preconditioning for the equation (1.1) with constant coefficients was analyzed in [5], [14] and [15], for example, where more general cases including convection-diffusion dominated flows were discussed. Hence our concern is to analyze the clustering 
situations of eigenvalues $\lambda$ and is to approximate extreme eigenvalues $\lambda$ of the semi-circulant preconditioned matrix $\hat{S}_{c}^{-1} \hat{L}_{v}$ which satisfy

$$
\lambda \hat{S}_{c} U=\hat{L}_{v} U
$$

where $U$ is the eigenvector corresponding to $\lambda$. This analysis can be done by using the derived formula of eigenvalues $\hat{\lambda}$ of the matrix $\hat{S}_{c}^{-1} \hat{L}_{c}$ satisfying

$$
\hat{\lambda} \hat{S}_{c} V=\hat{L}_{c} V,
$$

which was analyzed in [15]. In this purpose, we represent the eigenvalues $\lambda$ in (1.17) as follows:

$$
\lambda=\frac{\left(\hat{L}_{v} U, U\right)}{\left(\hat{S}_{c} U, U\right)}=\frac{\left(\hat{L}_{v} U, U\right)}{\left(\hat{L}_{c} U, U\right)} \cdot \frac{\left(\hat{L}_{c} U, U\right)}{\left(\hat{S}_{c} U, U\right)},
$$

where the $l^{2}$ inner product is defined by

$$
(U, V)=\sum_{k=1}^{N} u_{k} \bar{v}_{k}
$$

for complex vectors $U=\left(u_{1}, \ldots, u_{N}\right)$ and $V=\left(v_{1}, \ldots, v_{N}\right)$. Hence, we first investigate the field of values:

$$
\frac{\left(\hat{L}_{v} U, U\right)}{\left(\hat{L}_{c} U, U\right)}
$$

which means the preconditioning $\hat{L}_{v}$ by finite difference matrix $\hat{L}_{c}$.

This paper consists as follows: In section 2, the finite difference preconditioning for one and two dimensional cases are discussed in terms of coefficients of given differential equations. Finally, the semi-circulant preconditioner is discussed in section 3 with some numerical evidences.

\section{Preconditioning by finite difference}

The aim of this section is to get the bounds of eigenvalues of the preconditioned finite difference matrix in terms of coefficients functions given in (1.1) even if a finite difference preconditioner is well known (see, for example, [17] and [18]). Because the analysis for the $2 D$ case can be derived by the way of analyzing the $1 D$ case, we first consider the following one-dimensional elliptic operators $L_{v}^{1}$ and $L_{c}^{1}$ defined by

$$
L_{v}^{1} u:=-u^{\prime \prime}+a(x) u^{\prime}+d(x) u \quad \text { in } \quad(0,1)
$$

and

$$
L_{c}^{1} u:=-u^{\prime \prime}+d_{c} u \quad \text { in }(0,1),
$$

where $d_{c}=\min d(x)$ is a positive constant. Further we assume in this section that $a^{\prime}(x)$ is a bounded function on $[0,1]$,

$$
\left\|a^{\prime}\right\|_{\infty}:=\max _{x \in[0,1]}\left|a^{\prime}(x)\right|<\infty \text { and } \max _{x \in[0,1]}\left|\frac{a(x) h}{2}\right|<1 .
$$


Now let $\left\{x_{i}\right\}_{i=0}^{N+1}$ be the uniform knots such that

$$
x_{i}:=i h, \quad \text { where } h=\frac{1}{N+1} .
$$

Denote $a_{i}=a\left(x_{i}\right)$ and $d_{i}=d\left(x_{i}\right)$ for each $i=1, \ldots, N$. Then the usual central finite-difference approximation to $(2.1)$ at each knot $x_{i}, i=1, \ldots, N$ is given by

$$
\left(L_{v}^{1} u\right)_{i}=-\frac{1}{h^{2}}\left[\alpha_{i} u_{i+1}-2 u_{i}+\beta_{i} u_{i-1}\right]+d_{i} u_{i}, \quad i=1, \ldots, N
$$

where

$$
\alpha_{i}=1-\frac{a_{i} h}{2}, \quad \beta_{i}=1+\frac{a_{i} h}{2} .
$$

The change of indices with the boundary conditions $u_{0}=u_{N+1}=0$ yields that

$$
\begin{aligned}
& \sum_{i=1}^{N}\left(L_{v}^{1} u\right)_{i} \bar{u}_{i} \\
= & -\frac{1}{h^{2}} \sum_{i=1}^{N}\left[\alpha_{i}\left(u_{i+1}-u_{i}\right)-\beta_{i}\left(u_{i}-u_{i-1}\right)\right] \bar{u}_{i}+\sum_{i=1}^{N} d_{i}\left|u_{i}\right|^{2} \\
= & -\frac{1}{h^{2}}\left[\sum_{i=1}^{N} \alpha_{i}\left(u_{i+1}-u_{i}\right) \bar{u}_{i}-\sum_{i=0}^{N-1} \beta_{i+1}\left(u_{i+1}-u_{i}\right) \bar{u}_{i+1}\right]+\sum_{i=1}^{N} d_{i}\left|u_{i}\right|^{2} \\
= & \frac{1}{h^{2}} \sum_{i=0}^{N}\left(u_{i+1}-u_{i}\right)\left(\beta_{i+1} \bar{u}_{i+1}-\alpha_{i} \bar{u}_{i}\right)+\sum_{i=1}^{N} d_{i}\left|u_{i}\right|^{2} .
\end{aligned}
$$

Since

we have

$$
\beta_{i+1} \bar{u}_{i+1}-\alpha_{i} \bar{u}_{i}=\left(\bar{u}_{i+1}-\bar{u}_{i}\right)+\frac{h}{2}\left(a_{i+1} \bar{u}_{i+1}+a_{i} \bar{u}_{i}\right),
$$

$$
\begin{aligned}
& \sum_{i=1}^{N}\left(L_{v}^{1} u\right)_{i} \bar{u}_{i} \\
= & \frac{1}{h^{2}} \sum_{i=0}^{N}\left[\left|u_{i+1}-u_{i}\right|^{2}+\frac{h}{2}\left(u_{i+1}-u_{i}\right)\left(a_{i+1} \bar{u}_{i+1}+a_{i} \bar{u}_{i}\right)+h^{2} d_{i}\left|u_{i}\right|^{2}\right] .
\end{aligned}
$$

As a result of (2.6), by taking $a(x)=0$ and $d(x)=d_{c}$, we have

$$
\sum_{i=1}^{N}\left(L_{c}^{1} u\right)_{i} \bar{u}_{i}=\frac{1}{h^{2}} \sum_{i=0}^{N}\left[\left|u_{i+1}-u_{i}\right|^{2}+h^{2} d_{c}\left|u_{i}\right|^{2}\right],
$$

which has real valued for any complex numbers $u_{1}, \ldots, u_{N}$.

For convenience, put

$$
x:=\sum_{k=0}^{N}\left|u_{k+1}-u_{k}\right|^{2}, \quad y_{v}:=h^{2} \sum_{k=0}^{N} d_{k}\left|u_{k}\right|^{2}, \quad y_{c}:=h^{2} \sum_{k=0}^{N} d_{c}\left|u_{k}\right|^{2}
$$


and

$$
z_{v}:=\frac{h}{2} \sum_{k=0}^{N}\left(u_{k+1}-u_{k}\right)\left(a_{k+1} \bar{u}_{k+1}+a_{k} \bar{u}_{k}\right)
$$

Note that for any complex numbers $u_{1}, \ldots, u_{N}$, the new variables $x, y_{v}$ and $y_{c}$ are positive real values, but $z_{v}$ is complex value. First of all, let us estimate $z_{v}$ in the following lemma.

Lemma 2.1. The following estimates hold:

$$
\left|\operatorname{Re} z_{v}\right| \leq \frac{\left\|a^{\prime}\right\|_{\infty}}{2 d_{c}} y_{c}
$$

and

$$
\left|z_{v}\right| \leq \frac{\|a\|_{\infty}}{2 \sqrt{d_{c}}}\left(x+y_{c}\right)
$$

Proof. Since $u_{0}=u_{N+1}=0$ and $a_{k+1}-a_{k}=h a^{\prime}\left(\xi_{k}\right)$ for some $\xi_{k} \in\left(x_{k}, x_{k+1}\right)$, $z_{v}$ can be written as

$$
\begin{aligned}
z_{v} & =\frac{h}{2} \sum_{k=0}^{N}\left(u_{k+1}-u_{k}\right)\left(a_{k+1} \bar{u}_{k+1}+a_{k} \bar{u}_{k}\right) \\
& =\frac{h}{2} \sum_{k=0}^{N}\left(a_{k} u_{k+1} \bar{u}_{k}-a_{k+1} u_{k} \bar{u}_{k+1}\right) \\
& =\frac{h}{2} \sum_{k=0}^{N}\left\{a_{k}\left(u_{k+1} \bar{u}_{k}-u_{k} \bar{u}_{k+1}\right)-\left(a_{k+1}-a_{k}\right) u_{k} \bar{u}_{k+1}\right\} \\
& =\frac{h}{2} \sum_{k=0}^{N}\left\{a_{k}\left(u_{k+1} \bar{u}_{k}-u_{k} \bar{u}_{k+1}\right)-h a^{\prime}\left(\xi_{k}\right) u_{k} \bar{u}_{k+1}\right\}
\end{aligned}
$$

Since $u_{k+1} \bar{u}_{k}-u_{k} \bar{u}_{k+1}$ is pure imaginary number,

$$
\operatorname{Re} z_{v}=-\frac{h^{2}}{2} \sum_{k=0}^{N} a^{\prime}\left(\xi_{k}\right) \operatorname{Re}\left(u_{k} \bar{u}_{k+1}\right)
$$

Using the fact $|\operatorname{Re} z| \leq|z|$ and Cauchy-Schwarz inequality, we have

$$
\left|\operatorname{Re} z_{v}\right| \leq \frac{h^{2}\left\|a^{\prime}\right\|_{\infty}}{2} \sum_{k=0}^{N}\left|u_{k}\right|\left|u_{k+1}\right| \leq \frac{h^{2}\left\|a^{\prime}\right\|_{\infty}}{2} \sum_{k=0}^{N}\left|u_{k}\right|^{2}=\frac{\left\|a^{\prime}\right\|_{\infty}}{2 d_{c}} y_{c} .
$$


For the estimation of $\left|z_{v}\right|$, Cauchy-Schwarz inequality applied to the first term of (2.10) and boundary conditions yield that

$$
\begin{aligned}
\left|z_{v}\right| & \leq \frac{h}{2} \sum_{k=0}^{N}\left|u_{k+1}-u_{k}\right|\left(\left|a_{k+1}\right|\left|u_{k+1}\right|+\left|a_{k}\right|\left|u_{k}\right|\right) \\
& \leq \frac{\|a\|_{\infty} h}{2} \sum_{k=0}^{N}\left|u_{k+1}-u_{k}\right|\left|u_{k+1}\right|+\frac{\|a\|_{\infty} h}{2} \sum_{k=0}^{N}\left|u_{k+1}-u_{k}\right|\left|u_{k}\right| \\
& \leq\|a\|_{\infty}\left(\sum_{k=0}^{N}\left|u_{k+1}-u_{k}\right|^{2}\right)^{1 / 2}\left(h^{2} \sum_{k=0}^{N}\left|u_{k}\right|^{2}\right)^{1 / 2} .
\end{aligned}
$$

Using the so called $\varepsilon$-inequality:

$$
a b \leq \frac{1}{2 \varepsilon} a^{2}+\frac{\varepsilon}{2} b^{2}
$$

we have

$$
\begin{aligned}
\left|z_{v}\right| & \leq \frac{\|a\|_{\infty}}{2}\left(\frac{1}{\sqrt{d_{c}}} \sum_{k=0}^{N}\left|u_{k+1}-u_{k}\right|^{2}+\sqrt{d_{c}} h^{2} \sum_{k=0}^{N}\left|u_{k}\right|^{2}\right) \\
& =\frac{\|a\|_{\infty}}{2 \sqrt{d_{c}}}\left(x+y_{c}\right) .
\end{aligned}
$$

These arguments complete the proof.

The above lemma will be used to estimate the preconditioning results for $L_{v}$. The point of the following proposition is to estimate the lower and upper bounds of field values of preconditioned system $L_{c}^{-1} L_{v}$ in terms of the coefficient functions $a(x), d(x)$ and $d_{c}$ as possible as we can. The choice of preconditioner using leading term was analyzed in detail in [18].

Proposition 2.1. Assume that (2.3) holds. Let $\hat{L}_{v}^{1}$ and $\hat{L}_{\mathrm{c}}^{1}$ be the matrix of the finite difference approximation corresponding to the operators $L_{v}^{1}$ and $L_{c}^{1}$ respectively. For any nonzero complex vector $U=\left(u_{1}, \ldots, u_{N}\right)^{t}$, the following estimates hold:

$$
1-\frac{\left\|a^{\prime}\right\|_{\infty}}{2 d_{c}} \leq \operatorname{Re}\left(\frac{\left(\hat{L}_{v}^{1} U, U\right)}{\left(\hat{L}_{c}^{1} U, U\right)}\right) \leq 1+\frac{\left\|a^{\prime}\right\|_{\infty}}{2 d_{c}}+\frac{d_{\max }}{d_{c}}
$$

and

$$
\left|\frac{\left(\hat{L}_{v}^{1} U, U\right)}{\left(\hat{L}_{c}^{1} U, U\right)}\right| \leq 1+\frac{d_{\max }}{d_{c}}+\frac{\|a\|_{\infty}}{2 \sqrt{d_{c}}}<\infty,
$$

where $d_{\max }$ is a nonnegative constant such that

$$
0 \leq d_{k}-d_{c} \leq d_{\max }, \text { for all } k=0,1, \ldots, N .
$$


Proof. Let

$$
W:=\frac{\left(\hat{L}_{v}^{1} U, U\right)}{\left(\hat{L}_{c}^{1} U, U\right)}
$$

Then

$$
W=\frac{x+y_{v}+z_{v}}{x+y_{c}}=\frac{x+y_{v}+\operatorname{Re} z_{v}}{x+y_{c}}+i \frac{\operatorname{Im} z_{v}}{x+y_{c}} .
$$

Note that

$$
0 \leq d_{k}-d_{c} \leq d_{\max }, \quad \text { for } k=0,1, \ldots, N
$$

for some constant $d_{\max }$. From Lemma 2.1, we have

$$
\left|\frac{\operatorname{Re} z_{v}}{x+y_{c}}\right| \leq \frac{\left\|a^{\prime}\right\|_{\infty}}{2 d_{c}} \frac{y_{c}}{x+y_{c}} \leq \frac{\left\|a^{\prime}\right\|_{\infty}}{2 d_{c}}
$$

and from (2.15)

$$
0 \leq \frac{y_{v}-y_{c}}{x+y_{c}} \leq \frac{d_{\max }}{d_{c}}
$$

Then the real part of $W$

$$
\operatorname{Re}(W)=\frac{x+y_{v}+\operatorname{Re} z_{v}}{x+y_{c}}=1+\frac{y_{v}-y_{c}}{x+y_{c}}+\frac{\operatorname{Re} z_{v}}{x+y_{c}},
$$

can be estimated as

$$
1-\frac{\left\|a^{\prime}\right\|_{\infty}}{2 d_{c}} \leq \operatorname{Re}(W) \leq 1+\frac{\left\|a^{\prime}\right\|_{\infty}}{2 d_{c}}+\frac{d_{\max }}{d_{c}},
$$

and the absolute value of $W$ is

$$
\begin{aligned}
|W| \leq \frac{x+y_{v}+\left|z_{v}\right|}{x+y_{c}} & \leq 1+\frac{y_{v}-y_{c}}{x+y_{c}}+\frac{\|a\|_{\infty}}{2 \sqrt{d_{c}}} \frac{x+y_{c}}{x+y_{c}} \\
& \leq 1+\frac{d_{\max }}{d_{c}}+\frac{\|a\|_{\infty}}{2 \sqrt{d_{c}}} .
\end{aligned}
$$

These arguments complete the proof.

Now, let's consider the operators $L_{v}$ and $L_{c}$ in (1.1) and (1.8) with (1.9). Throughout this paper, we assume that $d(x, y)$ in (1.1) is a positive bounded function with $d_{c}=\min d(x, y)>0$ and further assume that $a_{x}(x, y)$ and $b_{y}(x, y)$ are bounded functions on $\Omega$, that is,

$$
\begin{aligned}
\left\|a_{x}\right\|_{\infty} & :=\max _{(x, y) \in \Omega}\left|a_{x}(x, y)\right|<\infty \text { and } \\
\left\|b_{y}\right\|_{\infty} & :=\max _{(x, y) \in \Omega}\left|b_{y}(x, y)\right|<\infty .
\end{aligned}
$$


Let us rewrite (1.3) as

$$
\begin{aligned}
& \left(L_{v} u\right)_{k, j} \\
= & -\frac{1}{h_{1}^{2}}\left[\left(1+\frac{a_{k, j} h_{1}}{2}\right) u_{k-1, j}-2 u_{k, j}+\left(1-\frac{a_{k, j} h_{1}}{2}\right) u_{k+1, j}\right]+\frac{d_{k, j}}{2} u_{k, j} \\
& -\frac{1}{h_{2}^{2}}\left[\left(1+\frac{b_{k, j} h_{2}}{2}\right) u_{k, j-1}-2 u_{k, j}+\left(1-\frac{b_{k, j} h_{2}}{2}\right) u_{k, j+1}\right]+\frac{d_{k, j}}{2} u_{k, j} .
\end{aligned}
$$

First we note that a similar one dimensional argument yields that

$$
\sum_{k, j=1}^{m_{1}, m_{2}}\left(L_{v} u\right)_{k, j} \bar{u}_{k, j}=I_{v}+I I_{v}
$$

where

$$
\begin{aligned}
I_{v}= & \frac{1}{h_{1}^{2}} \sum_{k, j=0}^{m_{1}, m_{2}}\left[\left|u_{k+1, j}-u_{k, j}\right|^{2}+\frac{h_{1}^{2}}{2} d_{k, j}\left|u_{k, j}\right|^{2}\right. \\
& \left.+\frac{h_{1}}{2}\left(u_{k+1, j}-u_{k, j}\right)\left(a_{k+1, j} \bar{u}_{k+1, j}+a_{k, j} \bar{u}_{k, j}\right)\right]
\end{aligned}
$$

and

$$
\begin{aligned}
I I_{v}= & \frac{1}{h_{2}^{2}} \sum_{k, j=0}^{m_{1}, m_{2}}\left[\left|u_{k, j+1}-u_{k, j}\right|^{2}+\frac{h_{2}^{2}}{2} d_{k, j}\left|u_{k, j}\right|^{2}\right. \\
& \left.+\frac{h_{2}}{2}\left(u_{k, j+1}-u_{k, j}\right)\left(b_{k, j+1} \bar{u}_{k, j+1}+b_{k, j} \bar{u}_{k, j}\right)\right] .
\end{aligned}
$$

For the preconditioning operator $L_{c}$, we have

$$
\sum_{k, j=1}^{m_{1}, m_{2}}\left(L_{c} u\right)_{k, j} \bar{u}_{k, j}=I_{c}+I I_{c}
$$

where

$$
I_{c}=\frac{1}{h_{1}^{2}} \sum_{k, j=0}^{m_{1}, m_{2}}\left[\left|u_{k+1, j}-u_{k, j}\right|^{2}+\frac{h_{1}^{2}}{2} d_{c}\left|u_{k, j}\right|^{2}\right]
$$

and

$$
I I_{c}=\frac{1}{h_{2}^{2}} \sum_{k, j=0}^{m_{1}, m_{2}}\left[\left|u_{k, j+1}-u_{k, j}\right|^{2}+\frac{h_{2}^{2}}{2} d_{c}\left|u_{k, j}\right|^{2}\right] .
$$

For the convenience, put

$$
I_{x}:=\sum_{k, j=0}^{m_{1}, m_{2}}\left|u_{k+1, j}-u_{k, j}\right|^{2}, I_{y, v}:=\frac{h_{1}^{2}}{2} \sum_{k, j=0}^{m_{1}, m_{2}} d_{k, j}\left|u_{k, j}\right|^{2}, I_{y, c}:=\frac{h_{1}^{2}}{2} \sum_{k, j=0}^{m_{1}, m_{2}} d_{c}\left|u_{k, j}\right|^{2},
$$


and

(2.27)

$I I_{x}:=\sum_{k, j=0}^{m_{1}, m_{2}}\left|u_{k, j+1}-u_{k, j}\right|^{2}, I I_{y, v}:=\frac{h_{2}^{2}}{2} \sum_{k, j=0}^{m_{1}, m_{2}} d_{k, j}\left|u_{k, j}\right|^{2}, I I_{y, c}:=\frac{h_{2}^{2}}{2} \sum_{k, j=0}^{m_{1}, m_{2}} d_{c}\left|u_{k, j}\right|^{2}$,

and

$$
I_{z, v}:=\frac{h_{1}}{2} \sum_{k, j=0}^{m_{1}, m_{2}}\left(u_{k+1, j}-u_{k, j}\right)\left(a_{k+1, j} \bar{u}_{k+1, j}+a_{k, j} \bar{u}_{k, j}\right)
$$

and

$$
I I_{z, v}:=\frac{h_{2}}{2} \sum_{k, j=0}^{m_{1}, m_{2}}\left(u_{k, j+1}-u_{k, j}\right)\left(b_{k, j+1} \bar{u}_{k, j+1}+b_{k, j} \bar{u}_{k, j}\right)
$$

Note that for any complex numbers $u_{1,1}, \ldots, u_{N, N}$, all these $I_{x}, I I_{x}, I_{y, v}, I I_{y, v}$ and $I_{y, c}, I I_{y, c}$ are positive real values, but $I_{z, v}$ and $I I_{z, v}$ only are complex values. First let us start with estimations for $I_{z, v}$ and $I I_{z, v}$ in the following lemma.

Lemma 2.2. The following estimates hold.

$$
\left|\operatorname{Re} I_{z, v}\right| \leq \frac{\left\|a_{x}\right\|_{\infty}}{d_{c}} I_{y, c}
$$

and

$$
\left|I_{z, v}\right| \leq \frac{\|a\|_{\infty}}{\sqrt{2 d_{c}}}\left(I_{x}+I_{y, c}\right)
$$

Also, it follows that

$$
\left|\operatorname{Re} I I_{z, v}\right|=\frac{\left\|b_{y}\right\|_{\infty}}{d_{c}} I I_{y, c}
$$

and

$$
\left|I I_{z, v}\right| \leq \frac{\|b\|_{\infty}}{\sqrt{2 d_{c}}}\left(I I_{x}+I I_{y, c}\right) .
$$

Proof. The similar arguments in the proof of Lemma 2.1 lead to the conclusions.

Let $\hat{L}_{v}$ and $\hat{L}_{c}$ denote the matrices of the finite difference approximations corresponding to the operators $L_{v}$ and $L_{c}$, respectively.

Proposition 2.2. Assume that (2.18) and (1.13) hold. For any nonzero complex vector $U=\left(u_{1,1}, \ldots, u_{m_{1}, m_{2}}\right)^{t}$, there are constants $c_{1}, c_{2}$ and $C$ independent of $N$ such that

$$
c_{1}:=1-\frac{\Gamma_{1}}{d_{c}} \leq \operatorname{Re}\left(\frac{\left(\hat{L}_{v} U, U\right)}{\left(\hat{L}_{c} U, U\right)}\right) \leq c_{2}:=1+\frac{\Gamma_{1}}{d_{c}}+\frac{2 d_{\max }}{d_{c}}
$$


and

$$
\left|\frac{\left(\hat{L}_{v} U, U\right)}{\left(\hat{L}_{c} U, U\right)}\right| \leq C:=1+\frac{2 d_{\max }}{d_{c}}+\frac{2 M}{\sqrt{2 d_{c}}}<\infty
$$

where $d_{\max }$ is a positive constant such that

$$
0 \leq d_{k, j}-d_{\mathcal{C}} \leq d_{\max } \text { for } 1 \leq k \leq m_{1}, 1 \leq j \leq m_{2},
$$

and

$$
\Gamma_{1}:=\max \left\{\left\|a_{x}\right\|_{\infty},\left\|b_{y}\right\|_{\infty}\right\}, \quad \text { and } \quad M:=\max \left\{\|a\|_{\infty},\|b\|_{\infty}\right\} .
$$

Proof. Let

$$
W:=\frac{\left(\hat{L}_{v} U, U\right)}{\left(\hat{L}_{c} U, U\right)}
$$

which can be rewritten as

$$
\begin{aligned}
W= & \frac{\varphi^{2}\left(I_{x}+I_{y, v}+I_{z, v}\right)+I I_{x}+I I_{y, v}+I I_{z, v}}{\varphi^{2}\left(I_{x}+I_{y, c}\right)+I I_{x}+I I_{y, c}} \\
= & \frac{\varphi^{2}\left(I_{x}+I_{y, v}\right)+I I_{x}+I I_{y, v}+\operatorname{Re}\left(\varphi^{2} I_{z, v}+I I_{z, v}\right)}{\varphi^{2}\left(I_{x}+I_{y, c}\right)+I I_{x}+I I_{y, c}} \\
& +i \frac{\operatorname{Im}\left(\varphi^{2} I_{z, v}+I I_{z, v}\right)}{\varphi^{2}\left(I_{x}+I_{y, c}\right)+I I_{x}+I I_{y, c}} .
\end{aligned}
$$

Note that the real part of $W$ can be written as

$$
\begin{aligned}
\operatorname{Re}(W) & =\frac{\varphi^{2}\left(I_{x}+I_{y, v}\right)+I I_{x}+I I_{y, v}+\operatorname{Re}\left(\varphi^{2} I_{z, v}+I I_{z, v}\right)}{\varphi^{2}\left(I_{x}+I_{y, c}\right)+I I_{x}+I I_{y, c}} \\
& =1+\frac{\varphi^{2}\left(I_{y, v}-I_{y, c}\right)+I I_{y, v}-I I_{y, c}}{\varphi^{2}\left(I_{x}+I_{y, c}\right)+I I_{x}+I I_{y, c}}+\frac{\operatorname{Re}\left(\varphi^{2} I_{z, v}+I I_{z, v}\right)}{\varphi^{2}\left(I_{x}+I_{y, c}\right)+I I_{x}+I I_{y, c}} \\
& \leq 1+\frac{I_{y, v}-I_{y, c}}{I_{y, c}}+\frac{I I_{y, v}-I I_{y, c}}{I I_{y, c}}+\frac{\operatorname{Re}\left(\varphi^{2} I_{z, v}+I I_{z, v}\right)}{\varphi^{2}\left(I_{x}+I_{y, c}\right)+I I_{x}+I I_{y, c}} .
\end{aligned}
$$

Using (2.30) and (2.32), we have

$$
\left|\frac{\operatorname{Re}\left(\varphi^{2} I_{z, v}+I I_{z, v}\right)}{\varphi^{2}\left(I_{x}+I_{y, c}\right)+I I_{x}+I I_{y, c}}\right| \leq \frac{\Gamma_{1}}{d_{c}} \frac{\varphi^{2} I_{y, c}+I I_{y, c}}{\varphi^{2}\left(I_{x}+I_{y, c}\right)+I I_{x}+I I_{y, c}} \leq \frac{\Gamma_{1}}{d_{c}} .
$$

Hence combining (2.39) with (2.40) yields that

$$
1-\frac{\Gamma_{1}}{d_{c}} \leq \operatorname{Re}(W) \leq 1+\frac{\Gamma_{1}}{d_{c}}+\frac{2 d_{\max }}{d_{c}} .
$$


Using (2.31) and (2.33), the estimates of absolute value of $W$ is as follows:

$$
\begin{aligned}
& |W| \leq \frac{\varphi^{2}\left(I_{x}+I_{y, v}\right)+I I_{x}+I I_{y, v}+\left|\varphi^{2} I_{z, v}+I I_{z, v}\right|}{\varphi^{2}\left(I_{x}+I_{y, c}\right)+I I_{x}+I I_{y, c}} \\
& \leq 1+\frac{\varphi^{2}\left(I_{y, v}-I_{y, c}\right)+I I_{y, v}-I I_{y, c}}{\varphi^{2}\left(I_{x}+I_{y, c}\right)+I I_{x}+I I_{y, c}} \\
& +\frac{\frac{\|a\|_{\infty}}{\sqrt{2 d_{c}}} \varphi^{2}\left(I_{x}+I_{y, c}\right)+\frac{\|b\|_{\infty}}{\sqrt{2 d_{c}}}\left(I I_{x}+I I_{y, c}\right)}{\varphi^{2}\left(I_{x}+I_{y, c}\right)+I I_{x}+I I_{y, c}} \\
& \leq 1+\frac{I_{y, v}-I_{y, c}}{I_{y, c}}+\frac{I I_{y, v}-I I_{y, c}}{I I_{y, c}}+\frac{\|a\|_{\infty}}{\sqrt{2 d_{c}}}+\frac{\|b\|_{\infty}}{\sqrt{2 d_{c}}} \\
& \leq 1+\frac{2 d_{\max }}{d_{c}}+\frac{2 M}{\sqrt{2 d_{c}}},
\end{aligned}
$$

where $M=\max \left\{\|a\|_{\infty},\|b\|_{\infty}\right\}$.

\section{Semi-circulant preconditioning}

In this section, we will provide the behaviors of eigenvalues of the semicirculant preconditioned matrix $\hat{S}_{c}^{-1} \hat{L}_{v}$ with some numerical evidences. First of all, let us recall the results in [15] for the semi-circulant preconditioned matrix $\hat{S}_{c}^{-1} \hat{L}_{c}$ for (1.8). We note that the eigenvalues of (1.18) has the $\left(m_{1} m_{2}-2 m_{1}\right)$ number of eigenvalues which are exactly 1 . The remaining $2 m_{1}$-number of the eigenvalues $\lambda$ are also real-valued. These results can be described as follows (see Theorem 5.3 and 5.5 in [15]):

Theorem 3.1. Let $\hat{d}_{j}=d_{c}-\frac{\tau_{j}}{h_{2}^{2}}$, where $\tau_{j}$ is the eigenvalues of $T_{1}$. Let

$$
M_{1}(j)=\sqrt{\hat{d}_{j}}, \quad M_{2}(j)=-\sqrt{\hat{d}_{j}}
$$

and

$K_{0}(j)=-\frac{\left[M_{1}(j) e^{M_{1}(j)}+M_{2}(j) e^{M_{2}(j)}\right]}{\left(e^{M_{1}(j)}-1\right)\left(1-e^{M_{2}(j)}\right)}, K_{1}(j)=\frac{M_{1}(j) e^{M_{1}(j)}-M_{2}(j) e^{M_{2}(j)}}{e^{M_{1}(j)}-e^{M_{2}(j)}}$

and

$$
\bar{s}=\bar{s}(j)=-2 \sqrt{\hat{d}_{j}}\left(\frac{e^{M_{1}}+e^{M_{2}}-2}{e^{M_{1}}-e^{M_{2}}}\right) .
$$

Let $\hat{\lambda}_{i}, i=1,2, \ldots, m_{1} m_{2}$, be the eigenvalues of (1.18) satisfying

$$
\hat{\lambda}_{1} \leq \hat{\lambda}_{2} \leq \cdots \leq \hat{\lambda}_{m_{1} m_{2}} .
$$

(a) The $m_{1}$-smallest eigenvalues $\hat{\lambda}_{j}$ satisfy

$$
.38<\frac{3-\sqrt{5}}{2} \leq \hat{\lambda}_{j} \leq 1, \quad j=1, \ldots, m_{1} .
$$


(b) There are the $\left(m_{1} m_{2}-2 m_{1}\right)$-number of eigenvalues $\hat{\lambda}_{j}$ which are exactly 1, i.e.,

$$
\hat{\lambda}_{j}=1, \quad j=m_{1}+1, \ldots, m_{1} m_{2}-m_{1} .
$$

(c) For the other $m_{1}$-number of the eigenvalues $\hat{\lambda}_{j}$, we have, as $h_{1}, h_{2} \rightarrow 0$, $\hat{\lambda}_{j} \approx \frac{1}{2}+\frac{2\left[K_{0}(j)+K_{1}(j)\right]}{\bar{s}(j)}+\frac{1}{h_{2}} \frac{2}{|\bar{s}(j)|}, \quad j=m_{1} m_{2}-m_{1}+1, \ldots, m_{1} m_{2}$.

Now, let us recall that the field of values $\mathcal{W}(A)$ of a given $n \times n$ matrix $A$ is defined as

$$
\mathcal{W}(A)=\left\{\frac{(A U, U)}{(U, U)}, \quad \mathbf{0} \neq U \in \mathbb{C}^{n}\right\} .
$$

Proposition 3.1. Assume that (2.18) and (1.13) hold. Suppose that the reaction coefficient $d(x, y)=d_{c}$ in (1.1) is constant which is chosen as sufficiently large enough so that

$$
\frac{\Gamma_{1}}{d_{c}} \text { and } \frac{M}{\sqrt{d_{c}}}
$$

are small enough, where $\Gamma_{1}$ and $M$ are defined in (2.37). Then the field values $\frac{\left(\hat{L}_{v} U, U\right)}{\left(\hat{L}_{c} U, U\right)}$ are clustered at number 1 . Hence the eigenvalues of $\hat{L}_{c}^{-1} L_{v}$ are also clustered at number 1.

Proof. This comes from Proposition 2.2.

Theorem 3.2. Assume that (2.18) and (1.13) hold. Suppose that $\left\{\hat{\lambda}_{i}\right\}_{i=1}^{m_{1} m_{2}}$ are the eigenvalues of $\hat{S}_{c}^{-1} \hat{L}_{c}$. Let $\left\{\lambda_{i}\right\}_{i=1}^{m_{1} m_{2}}$ be the eigenvalues of the semi-circulant preconditioned matrix $\hat{S}_{c}^{-1} \hat{L}_{v}$. Then we have

$$
\operatorname{Re}\left(w_{1}\right) \hat{\lambda}_{\min } \leq \operatorname{Re}\left(\lambda_{i}\right) \leq \operatorname{Re}\left(w_{2}\right) \hat{\lambda}_{\max }
$$

and

$$
\left|\lambda_{i}\right| \leq\left|w_{3}\right| \hat{\lambda}_{\max }
$$

as $h_{1}, h_{2} \rightarrow 0$, where $w_{i}^{\prime} s$ are constants in $\mathcal{W}\left(\hat{L}_{c}^{-1} \hat{L}_{v}\right)$ which satisfy, due to Proposition 2.2,

$$
\operatorname{Re}\left(w_{i}\right) \geq c_{1}, \quad \text { and } \quad\left|w_{i}\right| \leq C, \quad i=1,2,3,
$$

where $c_{1}$ and $C$ are independent of $h_{1}$ and $h_{2}$, and $\hat{\lambda}_{\min }$ and $\hat{\lambda}_{\max }$ are minimum and maximum of $\left\{\hat{\lambda}_{i}\right\}$, respectively.

Proof. First note that $\hat{L}_{c}^{-1} \hat{S}_{c}$ is symmetric and positive definite. Note that

$$
\frac{\left(\hat{L}_{v} U, U\right)}{\left(\hat{S}_{c} U, U\right)}=\frac{\left(\hat{L}_{c} U, U\right)}{\left(\hat{S}_{c} U, U\right)} \frac{\left(\hat{L}_{v} U, U\right)}{\left(\hat{L}_{c} U, U\right)} .
$$

Now the conclusions come from combining Theorem 3.1 and Proposition 2.2. 
Let us point out some special case of the above theorem. Assume that $a(x, y)=f(y), b(x, y)=g(x)$ and $d(x, y)=d_{c}$, so that $a_{x}=b_{y}=0$ and $d(x, y)$ is constant. Then we have $\Gamma_{1}=0$ and $d_{\max }=0$. Then, as $h_{1}, h_{2} \rightarrow 0$ we have from Proposition 2.2, for all $w \in \mathcal{W}\left(\hat{L}_{c}^{-1} \hat{L}_{v}\right)$,

$$
\operatorname{Re}(w)=1 \text { and }|w| \leq 1+\frac{2 M}{\sqrt{2 d_{c}}}
$$

Hence if we choose $d_{c}$ so that

$$
\lim \frac{M}{d_{c}} \rightarrow 0
$$

then

$$
\lim _{\frac{M}{d_{c}} \rightarrow 0} w=1 \text {. }
$$

These situations yield that all field values of $\hat{L}_{c}^{-1} \hat{L}_{v}$ cluster around 1 , so that all its eigenvalues cluster around 1 . Hence one may prove that $\lambda_{i} \sim \hat{\lambda}_{i}$. Summarizing the above notice, we will put as a theorem here

Theorem 3.3. Under assumption of Proposition 3.1, the eigenvalues $\left\{\hat{\lambda}_{i}\right\}_{i=1}^{m_{1} m_{2}}$ of $\hat{S}_{c}^{-1} \hat{L}_{c}$ are clustered at number 1 as $h_{1}$ and $h_{2}$ approach to 0 .

Proof. This result comes from combining Proposition 3.1 and Theorem 3.2.

Example 1. Consider

$$
L_{v} u=-\Delta u+y^{2} u_{x}+x^{3} u_{y}+\left(d_{c}+e^{x+y}\right) u
$$

with

$$
L_{c} u=-\Delta u+\left(d_{c}+1\right) u .
$$

We compute the eigenvalues $\hat{S}_{c}^{-1} \hat{L}_{v}$ for the cases $d_{c}=10$ and 10000 with mesh size $h_{1}=h_{2}=\frac{1}{40}$, which shows that its eigenvalues are clustering at a number 1 in Figure 3.1 as a constant $d_{c}$ is chosen large enough. These phenomena support Theorem 3.3.

Example 2. Consider

$$
L_{v} u=-\Delta u+e^{x y} u_{x}+e^{(x+y)} u_{y}+\left(d_{c}+x\right)\left(d_{c}+y\right) u
$$

with

$$
L_{c} u=-\Delta u+d_{c}^{2} u \text {. }
$$

We plot the eigenvalues of in Figure 3.2 for $h_{1}=h_{2}=\frac{1}{40}$. From these computations, we see that the eigenvalues of $\hat{S}_{c}^{-1} \hat{L}_{v}$ become clustering at a number 1 as $d_{c}$ increases. These phenomena support also Theorem 3.3.

Example 3. Consider the boundary value problem

$$
L_{v} u=-\Delta u+\cos (8 x y) u_{x}+\sin (x+y) u_{y}+d_{c} u=f(x, y) \quad \text { in } \Omega
$$

and

$$
L_{c} u=-\Delta u+d_{c} u
$$



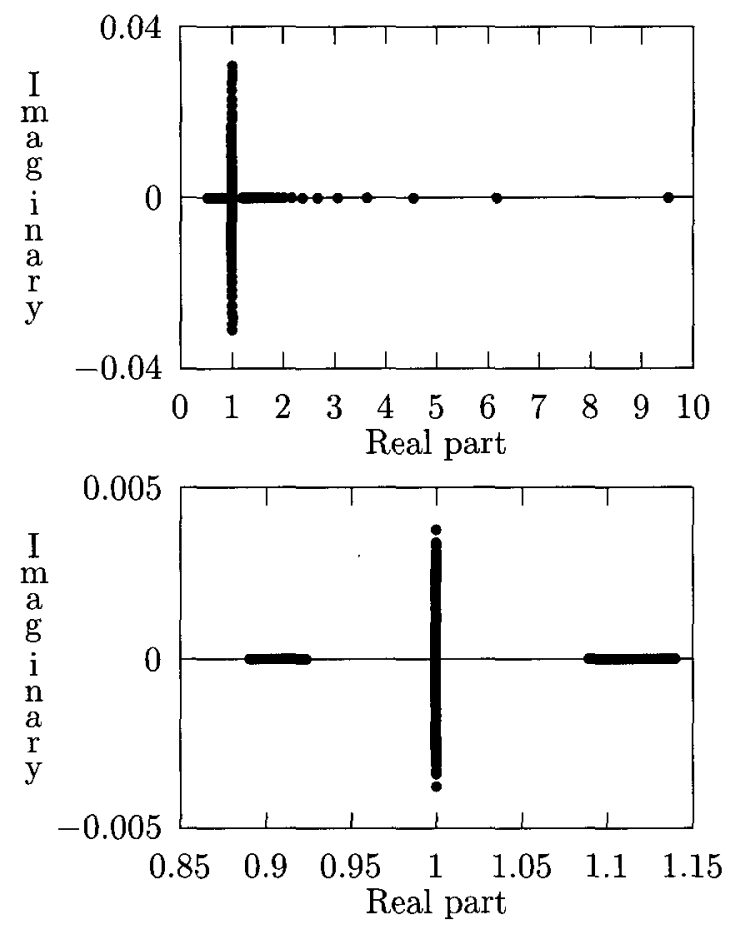

FIGURE 3.1. Distribution of the eigenvalues of $\hat{S}_{c}^{-1} \hat{L}_{v}$ for $d_{c}=$ 10 (Left) and $d_{c}=10000$ (Right)

with homogeneous Dirichlet boundary conditions, where the function $f(x, y)$ is chosen as the exact solution to be $u(x, y)=\sin (2 \pi x) \sin (2 \pi y)$. We compare the semi-circulant preconditioning system with no-preconditioning system in the view of the iteration number of the iterative method and maximum error of the exact solution in Table 3.1 and 3.2. We use the Bi-CGSTAB method.

We plot the eigenvalues of in Figure 3.3 for $h_{1}=h_{2}=\frac{1}{40}$. From these computations, we see that the eigenvalues of $\hat{S}_{c}^{-1} \hat{L}_{v}$ become clustering at a number 1 as $d_{c}$ increases as we have shown in Example 1 and 2.

\section{Concluding remark}

In the present paper, we analyzed the distribution of eigenvalues of a semicirculant preconditioned matrix based on the leading term and reaction term of the target elliptic operators (1.1) in which the coefficient of reaction term is chosen as the minimum of the given reaction coefficient function. Because of the equivalence of two finite difference matrices in the sense of $l^{2}$-inner product, it may be enough to set up a semi-circulant preconditioner having constant 

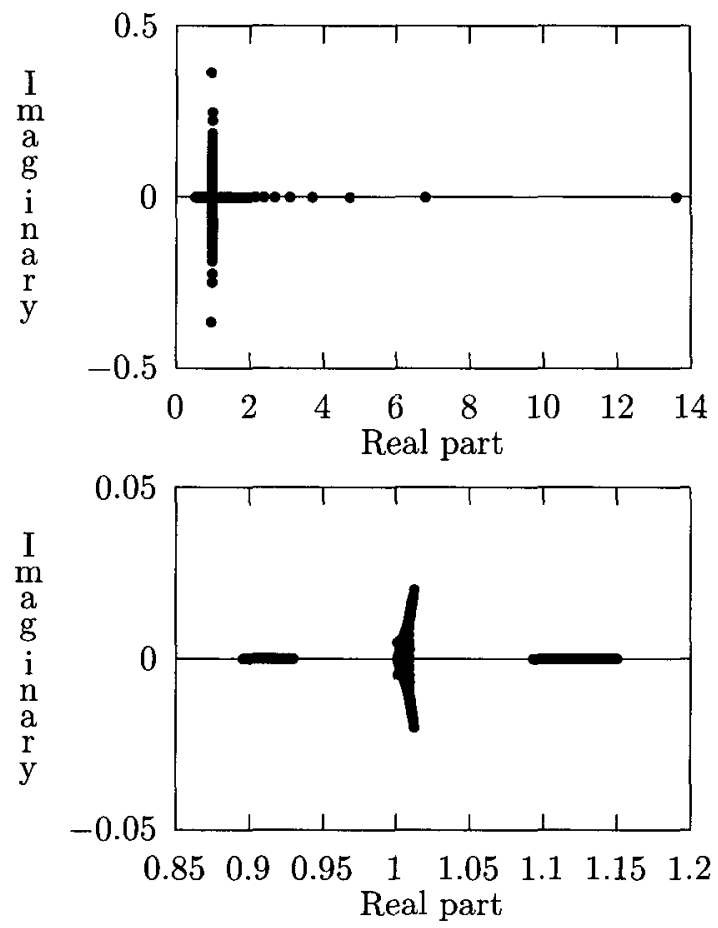

FigurE 3.2. Distribution of the eigenvalues of $\hat{S}_{c}^{-1} \hat{L}_{v}$ for $d_{c}=$ 1 (Left) and $d_{c}=100$ (Right)

\begin{tabular}{|c|c|c|c||c|c|c|}
\hline & \multicolumn{3}{|c||}{ Preconditioning } & \multicolumn{3}{c|}{ nopreconditioning } \\
\hline $\mathrm{N}$ & No. of Iter & $\begin{array}{c}\text { Maximum } \\
\text { Error }\end{array}$ & $\begin{array}{c}\text { CPU } \\
\text { time }\end{array}$ & No. of Iter & $\begin{array}{c}\text { Maximum } \\
\text { Error }\end{array}$ & $\begin{array}{c}\text { CPU } \\
\text { time }\end{array}$ \\
\hline 4 & 7 & 0.242661 & 1 & 8 & 0.242661 & 1 \\
8 & 10 & 0.055644 & 1 & 21 & 0.055644 & 1 \\
12 & 12 & 0.024340 & 1 & 36 & 0.024340 & 2 \\
16 & 13 & 0.013615 & 1 & 48 & 0.013615 & 2 \\
24 & 15 & 0.006027 & 4 & 73 & 0.006027 & 15 \\
32 & 17 & 0.003386 & 13 & 96 & 0.003386 & 66 \\
40 & 18 & 0.002165 & 33 & 126 & 0.002165 & 208 \\
\hline
\end{tabular}

TABLE 3.1. Number of iteration and maximum error for $d_{c}=1$

coefficients of the appropriate second-order elliptic operator. For several computational results, we certify that the eigenvalues of the preconditioned matrix are clustering at a number 1 and the preconditioned system are applicable to 

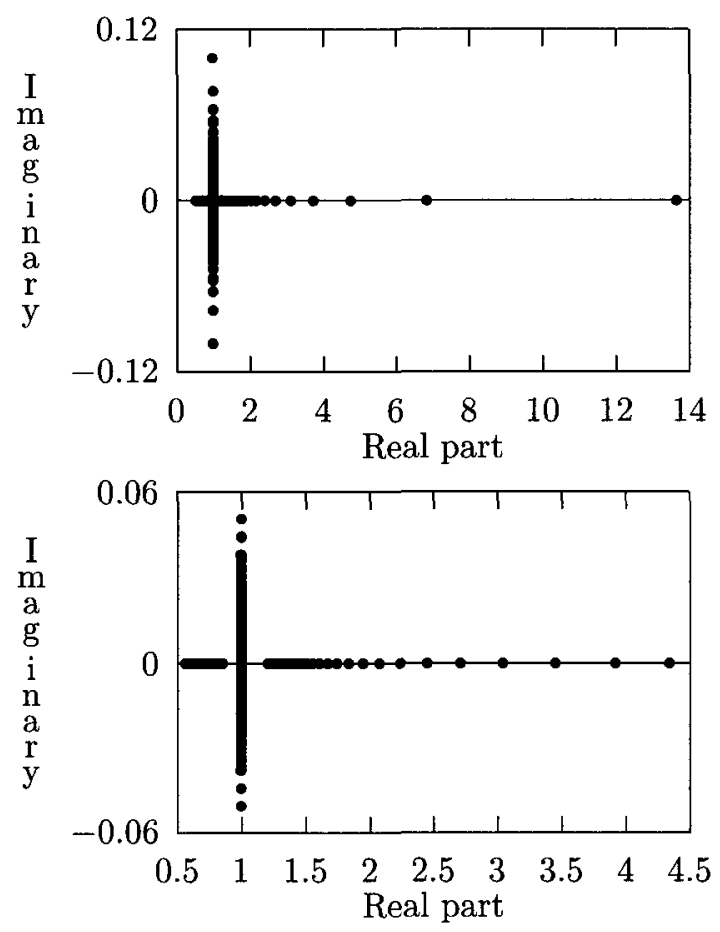

FIGURE 3.3. Distribution of the eigenvalues of $\hat{S}_{c}^{-1} \hat{L}_{v}$ for $d_{c}=$ 1 (Left) and $d_{c}=100$ (Right)

\begin{tabular}{|c|c|c|c||c|c|c|}
\hline & \multicolumn{3}{|c||}{ Preconditioning } & \multicolumn{3}{c|}{ nopreconditioning } \\
\hline $\mathrm{N}$ & No. of Iter & $\begin{array}{c}\text { Maximum } \\
\text { Error }\end{array}$ & $\begin{array}{c}\text { CPU } \\
\text { time }\end{array}$ & No. of Iter & $\begin{array}{c}\text { Maximum } \\
\text { Error }\end{array}$ & $\begin{array}{c}\text { CPU } \\
\text { time }\end{array}$ \\
\hline 4 & 4 & 0.093259 & 1 & 7 & 0.093259 & 1 \\
8 & 6 & 0.023517 & 1 & 14 & 0.023517 & 1 \\
12 & 7 & 0.010464 & 1 & 22 & 0.010464 & 2 \\
16 & 9 & 0.005888 & 2 & 30 & 0.005888 & 2 \\
24 & 11 & 0.002618 & 3 & 45 & 0.006027 & 10 \\
32 & 12 & 0.001473 & 9 & 69 & 0.001473 & 48 \\
40 & 13 & 0.000943 & 24 & 80 & 0.000943 & 133 \\
\hline
\end{tabular}

TABLE 3.2. Number of iteration and maximum error for $d_{c}=100$

the Bi-CGSTAB iterative method. Further, we hope to investigate the easy method to find the inverse of the semi-circulant preconditioning matrix. 


\section{References}

[1] Z.-Z. Bai, O. Axelsson, and S.-X. Qiu, A class of nested iteration schemes for linear systems with a coefficient matrix with a dominant positive definite symmetric part, Numer. Algorithms 35 (2004), no. 2-4, 351-372.

[2] Z.-Z. Bai, G. H. Golub and M. K. Ng, Hermitian and skew-Hermitian splitting methods for non-Hermitian positive definite linear systems, SIAM J. Matrix Anal. Appl. 24 (2003), no. 3, 603-626.

[3] Z.-Z. Bai, G. H. Golub, L.-Z. Lu, and J.-F. Yin, Block triangular and skew-Hermitian splitting methods for positive-definite linear systems, SIAM J. Sci. Comput. 26 (2005), no. $3,844-863$.

[4] D. Bertaccini, G. H. Golub, S. Serra Capizzano, and C. T. Possio, Preconditioned HSS methods for the solution of non-Hermitian positive definite linear systems and applications to the discrete convection-diffusion equation, Numer. Math. 99 (2005), no. 3, 441-484.

[5] R. Chan and T. Chan, Circulant preconditioners for elliptic problems, J. Numer. Linear Algebra Appl. 1 (1992), no. 1, 77-101.

[6] R. Chan and X. Jin, A family of block preconditioners for block systems, SIAM J. Sci. Statist. Comput. 13 (1992), no. 5, 1218-1235.

[7] R. Chan and M. K. Ng, Conjugate gradient methods for Toeplitz systems, SIAM Rev. 38 (1996), no. 3, 427-482.

[8] W. M. Cheung and M. K. Ng, Block-circulant preconditioners for systems arising from discretization of the three-dimensional convection-diffusion equation, Proceedings of the 9th International Congress on Computational and Applied Mathematics (Leuven, 2000), J. Comput. Appl. Math. 140 (2002), no. 1-2, 143-158.

[9] H. C. Elman and G. H. Golub, Iterative methods for cyclically reduced nonselfadjoint linear systems, Math. Comp. 54 (1990), no. 190, 671-700.

[10] _ Iterative methods for cyclically reduced nonselfadjoint linear systems. II., Math. Comp. 56 (1991), no. 193, 215-242.

[11] G. H. Golub, C. Grief, and J. M. Varah, Block orderings for tensor-product grids in two and three dimensions, Numer. Algorithms 30 (2002), no. 2, 93-111.

[12] G. H. Golub and D. Vanderstraeten, On the preconditioning of matrices with skewsymmetric splittings, Mathematical journey through analysis, matrix theory and scientific computation (Kent, OH, 1999), Numer. Algorithms 25 (2000), no. 1-4, 223-239.

[13] K. E. Gustafson and D. K. M. Rao, Numerical range, The field of values of linear operators and matrices. Universitext. Springer-Verlag, New York, 1997.

[14] L. Hemmingsson, A semi-circulant preconditioner for the convection-diffusion equation, Numer. Math. 81 (1998), no. 2, 211-248.

[15] S. D. Kim and S. V. Parter, Semicirculant preconditioning of elliptic operators, SIAM J. Numer. Anal. 41 (2003), no. 2, 767-795.

[16] A. R. Mitchell and D. F. Griffiths, The finite difference method in partial differential equations, A Wiley-Interscience Publication. John Wiley \& Sons, Ltd., Chichester, 1980.

[17] T. A. Manteuffel and J. Otto, Optimal equivalent preconditioners, SIAM J. Numer. Anal. 30 (1993), no. 3, 790-812.

[18] T. A. Manteuffel and S. V. Parter, Preconditioning and boundary conditions, SIAM J. Numer. Anal. 27 (1990), no. 3, 656-694.

[19] M. K. Ng, Circulant and skew-circulant splitting methods for Toeplitz systems, Proceedings of the 6th Japan-China Joint Seminar on Numerical Mathematics (Tsukuba, 2002). J. Comput. Appl. Math. 159 (2003), no. 1, 101-108. 
Hoi SUB KIM

Department of Mathematics and Information

KYUNGWON UNIVERSITY

Songnam 461-701, Korea

E-mail address: hskimm@kyungwon.ac.kr

SANG DONG KIM

DEPARTMENT OF MATHEMATICS

KYUNGPOOK NATIONAL UNIVERSITY

DAEgu 702-701, KoreA

E-mail address: skim@knu.ac.kr

YONG HUN LEE

Division of Mathematics and Statistical Informatics (INSTITUTE OF APPLiEd STATISTICS)

Chonbuk National UNIVERSITY

CHONJU 561-756, Korea

E-mail address: 1yh229@chonbuk.ac.kr 
